\title{
ASOCIACIÓN ENTRE LA PRÁCTICA DEPORTIVA FAMILIAR Y LA HABILIDAD COGNITIVA DEL ALUMNADO
}

\author{
Beatriz Berrios Aguayo ${ }^{1}$ \\ Pedro Ángel Latorre Román \\ Antonio Pantoja Vallejo \\ Universidad de Jaén. Jaén, España.
}

\begin{abstract}
Resumen. El propósito de este estudio fue observar la relación entre la práctica deportiva de los padres y la realizada por sus hijos con el rendimiento académico y la habilidad cognitiva en niños y niñas de Educación Primaria. Los datos fueron recogidos de dos escuelas de Educación Primaria de una ciudad del sur de España, una pública y otra concertada. Un total de 308 niños de 8 a 12 años (edad $=9,72 \pm 1,25$ años de edad), participaron en el estudio. Se utilizó un cuestionario sociodemográfico mediante el cual se recabó la información sobre la práctica deportiva, se pidieron las notas del alumnado al centro para analizar el rendimiento académico y se utilizó el instrumento Goodenough-Harris test cogiendo la puntuación bruta de éste para analizar la habilidad cognitiva. Se encontró correlación positiva entre la práctica deportiva de los padres y la realizada por sus hijos $(\mathrm{p}<, 001)$. También se obtuvieron resultados positivos en la asociación de la práctica deportiva del alumnado con su rendimiento académico $(\mathrm{p}<, 001)$ y su habilidad cognitiva $(\mathrm{p}<, 000)$. En conclusión, se confirma la existencia de una relación entre la mayor práctica deportiva realizada por los padres y la de sus hijos que a la vez influye en el rendimiento académico y la habilidad cognitiva del alumnado sugiriendo con esto que un aumento de la práctica deportiva de los padres y los hijos proporcionaría beneficios cognitivos.
\end{abstract}

Palabras clave: Deporte, rendimiento académico, habilidad cognitiva, niños, Educación Primaria

\section{ASSOCIATION BETWEEN FAMILY SPORT AND COGNITIVE ABILITY OF STUDENTS}

\begin{abstract}
The purpose of the study was to observe the relationship between parents' sport practice and their children's sport practice. We also compare children's sport practice with their academic achievement and cognitive ability. Data were collected from two schools of primary education in southern Spain. A total of 308 children aged 8 to 12 years (mean age $=9.72 \pm 1.25$ years) volunteered to participate. A socio-demographic questionnaire was used to collected sport practice, student's grades were asked to the academic center, and the Goodenough-Harris test was used to analyze their cognitive ability. Positive correlation between parents' sport practice and their children's was found $(\mathrm{p}<0.001)$. Also positive correlation was found between children's sport practice, their academic performance and their cognitive ability. In conclusion, the results confirm the relationship between a higher parent's sport
\end{abstract}

\footnotetext{
${ }^{1}$ Correspondencia: Beatriz Berrios Aguayo. Universidad de Jaén.bba00005@red.ujaen.es.
} 
practice and their children's and that both simultaneously influence in children's academic performance and cognitive ability.

Key words: Sport, academic performance, cognitive ability, children, Elementary school.

\title{
ASSOCIAÇÃO ENTRE A PRÁTICA DESPORTIVA FAMILIAR E A HABILIDADE COGNITIVA DOS ESTUDANTES
}

\begin{abstract}
Resumo. O propósito deste estudo foi observar a relação entre a prática desportiva dos pais e a realizada por seus filhos com o rendimento escolar e a habilidade cognitiva em meninos e meninas do Ensino Fundamental. Os dados foram recolhidos de duas escolas de Ensino Fundamental de uma cidade do sul da Espanha, uma pública e outra privada. Um total de 308 estudantes de 8 a 12 anos (idade $=9,72 \pm 1,25$ anos de idade) participaram do estudo. Foi utilizado um questionário sociodemográfico para recolher dados sobre a prática desportiva, foram solicitadas ao centro escolar as notas para analisar o rendimento e utilizado o instrumento Goodenough-Harris test, considerando a pontuação bruta deste para analisar a habilidade cognitiva. Foi encontrada correlação positiva entre a prática desportiva dos país e a realizada pelos filhos $(\mathrm{p}<, 001)$. Também foram obtidos resultados positivos na associação da prática desportiva dos estudantes com o rendimento escolar $(\mathrm{p}<, 001)$ e sua habilidade cognitiva $(\mathrm{p}<, 000)$. $\mathrm{O}$ estudo confirma a existência de uma relação entre a maior prática desportiva realizada pelos país e a de seus filhos e que, por sua vez, influencia no rendimento escolar e na habilidade cognitiva das crianças, sugerindo que um aumento da prática desportiva dos pais e dos filhos proporciona benefícios cognitivos.
\end{abstract}

Palavras-chave: Desporto, rendimento escolar, habilidade cognitiva, crianças, Ensino Fundamental.

\section{Introducción}

En los últimos años, se ha podido observar, a partir de diferentes investigaciones como las de Ellemberg y Louis-Deschênes (2010) y Gallotta et al. (2014), los beneficios que tiene la práctica de actividad física en cuanto al desempeño académico y cognitivo. La práctica de actividad física promueve mejoras en ciertos procesos cognitivos (Hernández y Portolés, 2016). A su vez, intervenciones en contextos escolares, realizando programas relacionados con un aumento de la práctica de actividad física han incrementado aspectos cognitivos en el alumnado (Janssen et al., 2014; Soga, Shishido, \& Nagatomi, 2015). Se puede comprobar una conexión entre el crecimiento del cuerpo y la condición física (Ortega et al., 2011; Travill, 2011) y la cognición (Heinonen et al., 2008).

Atendiendo a las afirmaciones expuestas anteriormente, se puede deducir a qué se refiere cuando se habla de condición física, considerando ésta como la capacidad o potencial físico de una persona (Devís \& Velert, 1992) que constituye un estado del organismo originado por el entrenamiento, es decir, por la repetición sistemática de ejercicios programados (Casterad, 2004). 
Por otro lado, según la Real Academia Española, se entiende por cognición el hecho de conocer, y por rendimiento académico la capacidad cognitiva que le permite al alumno hacer una elaboración mental de las implicaciones, consiguiendo las autopercepciones de sus habilidades y esfuerzos (Navarro, 2003).

Se puede determinar que la razón de esa relación existente entre la condición física del alumnado y su desarrollo cognitivo es cuando se ponen en juego el cerebelo y la corteza prefrontal en ambas (Diamond, 2000). Los niños que están en buena forma física presentan una mayor activación cortical y por tanto un mayor rendimiento cognitivo (Tomporowski, Davis, Miller, \& Naglieri, 2008). En este sentido, la condición física, puede beneficiar la cognición en los períodos anteriores de la vida que se caracterizan por el desarrollo y la organización del sistema nervioso (Hillman, Buck, Themanson, Pontifex, \& Castelli, 2009).

Cuando se trata de evaluar el rendimiento académico, se analizan en mayor o menor grado los factores que pueden determinar su adquisición; generalmente se consideran, entre otros, factores socioeconómicos, la amplitud de los programas de estudio, las metodologías de enseñanza utilizadas, la dificultad de emplear una educación personalizada, los conceptos previos que tienen los alumnos, así como el nivel de pensamiento formal de los mismos (Benitez, Gimenez y Osicka, 2000).

Atendiendo a esa multifactorialidad a la hora de definir las causas de un adecuado o inadecuado rendimiento académico, se puede observar cómo, algunas investigaciones, como la de Navarro (2003), determinan que existen otros factores influyentes en el nivel de rendimiento académico. La práctica deportiva de los padres aumenta la probabilidad de que sus hijos realicen algo parecido (Suárez \& Parra, 2005). Los resultados de algunos estudios demuestran que hay diferencias estadísticamente significativas a favor de los niños en cuanto a la práctica deportiva. Del mismo modo, el hábito de practicar actividad física frecuentemente, tanto el padre como la madre del joven, favorece una mayor participación deportiva por parte del escolar (Andújar, 2001). La finalidad de que los padres realicen dicha actividad y por lo tanto que sus hijos la realicen también, es los beneficios cognitivos que la actividad física tiene en los niños que las practican.

A raíz de lo expuesto con anterioridad, se establece como hipótesis de la investigación, que una mayor práctica deportiva de los padres se asocia con una mayor práctica deportiva de los hijos llevando a estos últimos a una mayor habilidad cognitiva y rendimiento académico.

El objetivo de la investigación consistió en determinar si la afirmación de la hipótesis era certera y por lo tanto la práctica deportiva paterna se asociaba con la del hijo y si, a su vez, esa práctica deportiva del hijo tenía relación con las variables cognitivas determinadas en la hipótesis realizando una discriminación por sexos. 


\section{Método}

\section{Diseño}

La investigación es un estudio transversal en la que los resultados son analizados en un momento determinado y a partir de ahí se obtienen unas conclusiones. En ella existe una estrecha relación e interacción entre nosotros y los participantes. La metodología se caracterizará por su carácter transformadora de la realidad educativa actual.

Los motivos expuestos anteriormente llevan también a la afirmación de que se trata de una investigación de tipo descriptiva-correlativa cuyo objetivo es observar la realidad, describirla y relacionar diferentes variables dependientes como el rendimiento académico y la habilidad cognitiva con variables independientes como la práctica deportiva del alumnado.

\section{Participantes}

Los participantes fueron 308 niños (edad $=9,72 \pm 1,25$ años de edad, rango de edad = 8-12 años), 156 niñas y 152 niños, pertenecientes a dos escuelas de Educación Primaria en la capital de Jaén. A los padres se les dio una descripción verbal explícita de la naturaleza y el propósito del estudio. De igual forma, se obtuvo su consentimiento para permitir la participación del alumnado. Los estudiantes con discapacidades intelectuales o físicas no participaron en este estudio. Más características de los participantes se muestran en la tabla 1. El estudio se realizó en cumplimiento de las normas de la Declaración de Helsinki (2013) y siguiendo las directrices de la Comunidad Europea para la Buena Práctica Clínica (111/3976/88 de julio de 1990), así como el marco legal español para la investigación clínica en humanos (Real Decreto 561/1993 sobre ensayos clínicos).

\section{Instrumentos y pruebas}

\section{Medidas antropométricas}

Se pidió a través de un cuestionario el peso de los padres. Las medidas antropométricas del alumnado se midieron en el centro escolar. La altura $(\mathrm{cm})$ se calculó con un estadiómetro (Seca 222, Hamburgo, Alemania) y el peso (kg) con una báscula (Seca 899, Hamburgo, Alemania). El índice de masa corporal (IMC) se calculó dividiendo el peso (en kilogramos) por la talla al cuadrado (en metros).

\section{Práctica deportiva}

En cuanto a la evaluación de la práctica deportiva de los padres y de los hijos se entregó un cuestionario sociodemográfico en el que se preguntaba si realizaban prácticas deportivas padres e hijos y el número de sesiones realizadas a la semana. Una vez realizado el cuestionario se analizaron intervalos entre padres que realizan varias sesiones a la semana junto a sus hijos, padres que realizan algunas y padres que no realizan ninguna práctica deportiva. 


\section{Capacidad cognitiva y rendimiento académico}

La habilidad cognitiva del alumnado se registró mediante el test GoodenoughHarris Dranwing (Harris, 1969) el cual informa de la edad mental del alumnado al igual que de su cociente intelectual (CI). En este test el niño debe de dibujar a un hombre y a una mujer de cuerpo entero. No se le da especificaciones de cómo debe realizar ambos dibujos, simplemente se le informa de que lo hagan lo mejor posible y como su imaginación le dicte. En esta prueba lo importante es que los participantes consigan dibujar el máximo número de detalles corporales en ambas figuras.

En cuanto al rendimiento académico, fue obtenido a través de las notas de las áreas de lengua, matemáticas, conocimiento del medio (nota media del área de ciencias naturales y ciencias sociales), inglés, educación física, plástica y música del último trimestre registrado. Es considerado el promedio de cada una de las asignaturas en el análisis de datos.

\section{Procedimiento}

Una vez obtenidos los permisos apropiados en las escuelas y los consentimientos informados de los padres, se procedió a la aplicación de pruebas y cuestionarios. Un investigador previamente entrenado realizó la evaluación. En primer lugar, se entregaron los cuestionarios sociodemográficos a los padres para que lo rellenasen con sus datos y los datos de sus hijos. En esos cuestionarios adquirimos medidas antropométricas de los padres y el conocimiento de la práctica o no deportiva tanto de los padres como de los hijos, además del número de sesiones en caso de ser realizada. Seguidamente se tomaron las medidas antropométricas del alumnado. Por último, se pasó el test Goodenough-Harris Dranwing (GHDT). Disponían del tiempo necesario, siendo la premisa fundamental la buena realización de los dibujos dentro de sus posibilidades. A su vez se le pidieron a la tutora las notas de las diferentes áreas del último trimestre realizado con el consentimiento de la dirección del centro y de los padres.

\section{Análisis estadístico}

La estadística descriptiva se representó como media (SD) y el porcentaje que se llevó a cabo en las variables de actividad física de los padres, deporte de los hijos, rendimiento académico, habilidad cognitiva, edad y sexo del alumnado. La prueba chicuadrado y análisis descriptivo se utilizó para determinar la relación entre la práctica deportiva de los padres y la de sus hijos. Una correlación de Pearson y un análisis de covarianza (Ancova) se llevaron a cabo para determinar la relación entre la práctica deportiva realizada por los padres, la realizada por los hijos y el rendimiento académico de éstos últimos. Para finalizar, se realizó otro análisis de covarianza entre el deporte practicado por el alumno y el rendimiento académico, utilizando la habilidad cognitiva como covariable. El nivel de significación fue de $\mathrm{p}<0.01$. Se realizó el análisis de datos con SPSS (versión 21, SPSS Inc., Chicago, Ill.) 


\section{Resultados}

\section{Actividad física realizada por los padres y por los hijos}

Los padres que realizan actividad física y que tienen hijos que practican algún deporte en horario extraescolar corresponde a un $84,2 \%$, mientras que un 55,3\% representa a aquellos padres que practican actividad física, pero sus hijos no realizan ningún deporte extraescolar, demostrando así, como se puede ver en la tabla 1, que la práctica de actividad física de los padres influye en la práctica deportiva del hijo.

Tabla 1. Resultados de la asociación entre la práctica deportiva de los padres y la práctica deportiva de los hijos.

\begin{tabular}{|c|c|c|c|c|}
\hline & & \multicolumn{2}{|c|}{ Deporte hijo } & \multirow[b]{2}{*}{ Total } \\
\hline & & Sí & No & \\
\hline \multirow[t]{4}{*}{ Actividad física Sí } & Recuento & 133,0 & 25,0 & 158,0 \\
\hline & $\begin{array}{l}\% \text { dentro de } \\
\text { actividad física }\end{array}$ & 84,2 & 15,8 & 100,0 \\
\hline & Recuento & 83,0 & 67,0 & 150,0 \\
\hline & $\begin{array}{l}\% \text { dentro de } \\
\text { actividad física }\end{array}$ & 55,3 & 44,7 & 100,0 \\
\hline \multirow[t]{2}{*}{ Total } & Recuento & 216,0 & 92,0 & 308,0 \\
\hline & $\begin{array}{l}\% \text { dentro de } \\
\text { actividad física }\end{array}$ & 70,1 & 29,9 & 100,0 \\
\hline
\end{tabular}

\section{Deporte hijo y sexo}

Un $80,9 \%$ de los niños realizan deporte en horario extraescolar frente a un $59,6 \%$ de las niñas que no lo realizan. Esto determina que la práctica deportiva es mayor en el género masculino que en el femenino en el alumnado de Educación Primaria. 
Tabla 2. Relación entre el deporte practicado por el alumnado y su sexo.

\begin{tabular}{|c|c|c|c|c|c|}
\hline & & & \multicolumn{2}{|c|}{ Deporte hijo } & \multirow{2}{*}{ Total } \\
\hline & & & Sí & No & \\
\hline \multirow[t]{4}{*}{ Sexo } & Niña & Recuento & 93,0 & 63,0 & 156 \\
\hline & & $\%$ dentro de sexo & 59,6 & 40,4 & 100,0 \\
\hline & Niño & Recuento & 123,0 & 29,0 & 152,0 \\
\hline & & $\%$ dentro de sexo & 80,9 & 19,1 & 100,0 \\
\hline \multirow[t]{2}{*}{ Total } & & Recuento & 216,0 & 92,0 & 308,0 \\
\hline & & $\%$ dentro de sexo & 70,1 & 29,9 & 100,0 \\
\hline
\end{tabular}

\section{Deporte hijo y promedio notas del alumnado}

Se encuentran diferencias significativas positivas en cuanto a la práctica deportiva del alumnado y el promedio de notas (tablas 3 y 4). El alumnado que realiza una mayor práctica deportiva a su vez tiene mejores puntuaciones académicas.

Tabla 3. Correlación entre la práctica deportiva de los padres y de los hijos y el rendimiento académicos.

\section{Actividad}

física Deporte hijo Promedio notas

\begin{tabular}{llrrr}
\hline Actividad física & Correlación de & 1 &, $315^{* *}$ &,- 082 \\
& Pearson & &, 000 &, 153 \\
& Sig. (bilateral) & 308 & 308 & 308 \\
Deporte hijo & $\mathrm{N}$ &, $315^{* *}$ & 1 &,$- 119^{*}$ \\
& Correlación de & & &, 036 \\
& Pearson &, 000 & & 308 \\
& Sig. (bilateral) & 308 & 308 & 1 \\
Promedio & $\mathrm{N}$ &,- 082 &,$- 119^{*}$ & \\
Notas & Correlación de &, 153 &, 036 & 308 \\
& Pearson & 308 & 308 & \\
\hline
\end{tabular}

Significatividad $=\mathrm{P}<0,01$ 
Tabla 4. Correlación entre el deporte practicado por el alumnado y el promedio notas.

\begin{tabular}{|c|c|c|c|c|c|c|}
\hline Origen & $\begin{array}{l}\text { Suma de } \\
\text { cuadrados }\end{array}$ & gl & $\begin{array}{c}\text { Cuadrático } \\
\text { promedio }\end{array}$ & $\mathrm{F}$ & Sig. & Eta \\
\hline Modelo corregido & $8,345^{\mathrm{a}}$ & 2 & 4,172 & 2,545 & ,080 & ,016 \\
\hline Interceptación & 13446,786 & 1 & 13446,786 & 8202,107 & ,000 & ,964 \\
\hline Deporte hijo & 4,960 & 1 & 4,960 & 3,025 &, 083 & ,010 \\
\hline Actividad física & 1,091 & 1 & 1,091 & ,666 & ,415 & ,002 \\
\hline Error & 500,026 & 305 & 1,639 & & & \\
\hline Total & 20060,411 & 308 & & & & \\
\hline Total corregido & 508,371 & 307 & & & & \\
\hline
\end{tabular}

$\mathrm{R}$ al cuadrado $=, 016(\mathrm{R}$ al cuadrado ajustada $=, 010)$

\section{Asociación entre el deporte realizado por el hijo, su habilidad cognitiva y su rendimiento académico}

Si se escoge como variable dependiente el promedio de notas, como variable independiente el deporte practicado por el alumnado y como covariable la habilidad cognitiva de éste, observamos que el deporte realizado por los niños influye en las puntuaciones académicas y en las puntuaciones con respecto a su habilidad cognitiva (tablas 5 y 6 )

Tabla 5. Asociación entre el deporte realizado por el niño y su rendimiento académico tomando como covariable la habilidad cognitiva.

\begin{tabular}{lrrrrrr}
\hline Origen & $\begin{array}{r}\text { Tipo III de suma } \\
\text { de cuadrados }\end{array}$ & Gl & $\begin{array}{r}\text { Cuadrático } \\
\text { promedio }\end{array}$ & F & Sig. & Eta \\
\hline Modelo corregido & $48,687^{\mathrm{a}}$ & 2 & 24,344 & 16,152 &, 000 &, 096 \\
Interceptación & 1216,824 & 1 & 1216,824 & 807,363 & $, 000,726$ \\
Habilidad & 41,434 & 1 & 41,434 & 27,492 & $, 000,083$ \\
cognitiva & 7,041 & 1 & 7,041 & 4,672 & $, 031,015$ \\
Deporte hijo & 459,684 & 305 & 1,507 & & & \\
Error & 20060,411 & 308 & & & & \\
Total & 508,371 & 307 & & & & \\
Total corregido & & & & & &
\end{tabular}

$\mathrm{R}$ al cuadrado $=, 016(\mathrm{R}$ al cuadrado ajustada $=, 010)$ 
Tabla 6. Análisis de la asociación entre el deporte realizado por el niño, el promedio de sus notas y su habilidad cognitiva.

\begin{tabular}{lllll}
\hline Deporte hijo & Media & $\begin{array}{l}\text { Error estándar } \\
\text { Límite inferior }\end{array}$ & $\begin{array}{l}\text { Intervalo de confianza al 95\% } \\
\text { Límite superior }\end{array}$ \\
\hline Sí & $8,066^{\mathrm{a}}$ &, 084 & 7,902 & 8,231 \\
No & $7,736^{\mathrm{a}}$ &, 128 & 7,484 & 7,988 \\
\hline
\end{tabular}

\section{La habilidad cognitiva del niño, el deporte practicado por él y la edad}

La práctica deportiva del alumnado influye en la habilidad cognitiva que va aumentando a su vez en relación con la edad cronológica. Aquellos niños que practican algún deporte en horario extraescolar obtienen mejores puntuaciones en el test de Goodenough-Harris Drawing Test (GHDT). Esto se puede observar en las figuras 1, 2, 3, 4, 5, 6, 7 y 8. Vemos cómo la calidad de los dibujos y el número de detalles empleados aumenta con respecto a la edad. Las figuras 1 y 2 corresponden a $3^{\circ}$ de Educación Primaria (EP), la 3 y 4 a $4^{\circ}$ EP, la 5 y 6 a $5^{\circ}$ EP y la 7 y 8 corresponden a $6^{\circ}$ EP. Los dibujos de las figuras corresponden a su vez a niños y niñas que practican deporte y obtuvieron una puntuación mayor que niños que no lo practican.

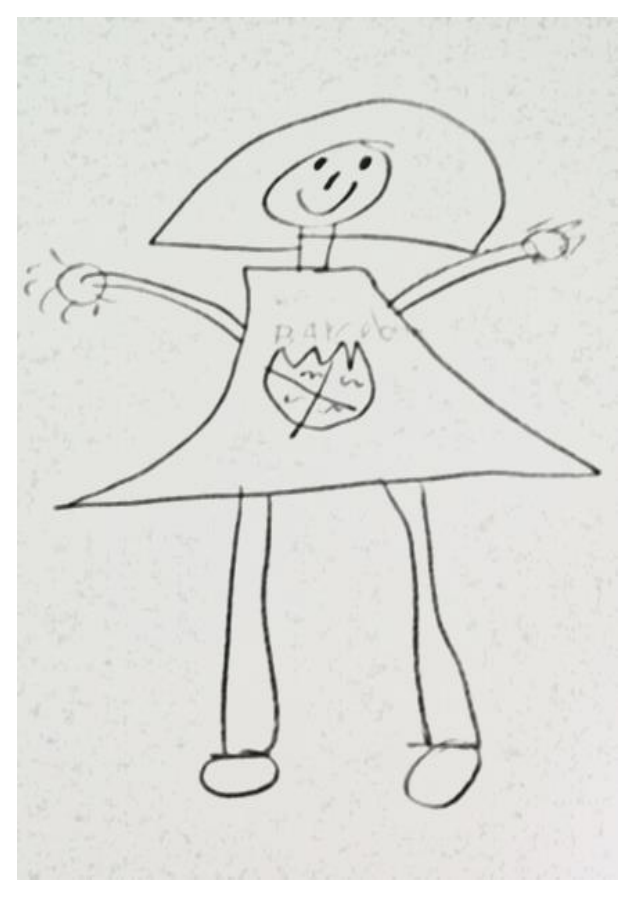

Figura 1. Dibujo de mujer. Niña $3^{\circ} \mathrm{EP}$ (Puntuación bruta test GHDT=22)

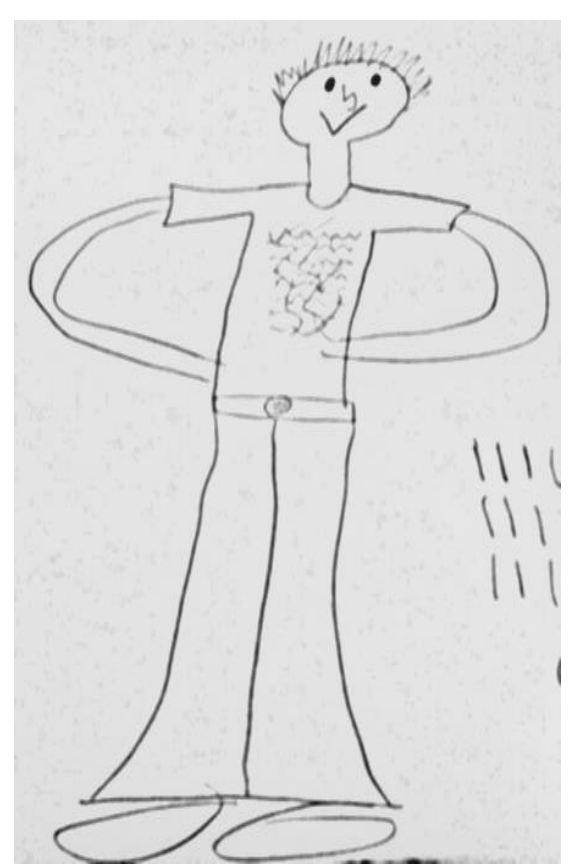

Figura 2. Dibujo de hombre. Niña $3^{\circ} \mathrm{EP}$ (Puntuación bruta test GHDT=20) 


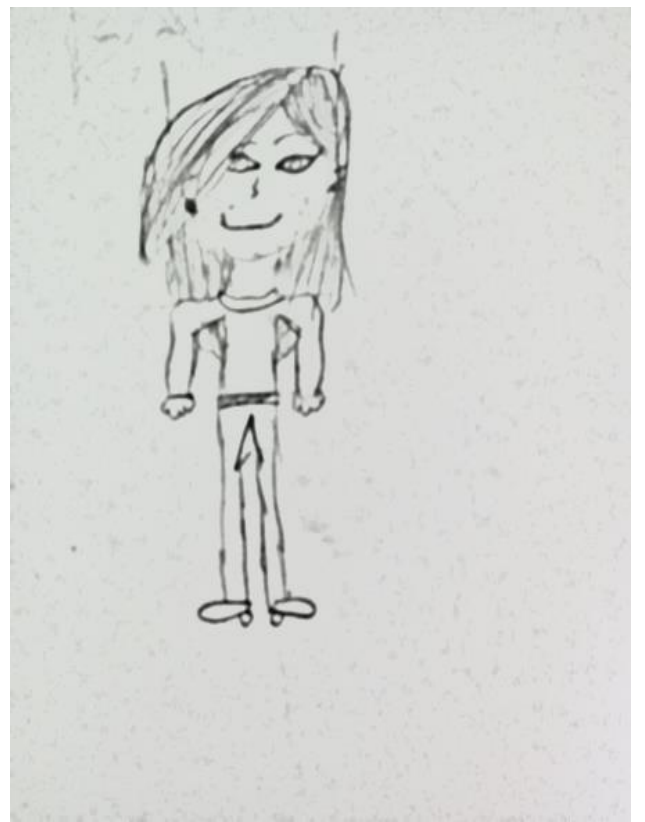

Figura 3. Dibujo de mujer. Niño $4^{\circ} \mathrm{EP}$ (Puntuación bruta test GHDT=28)

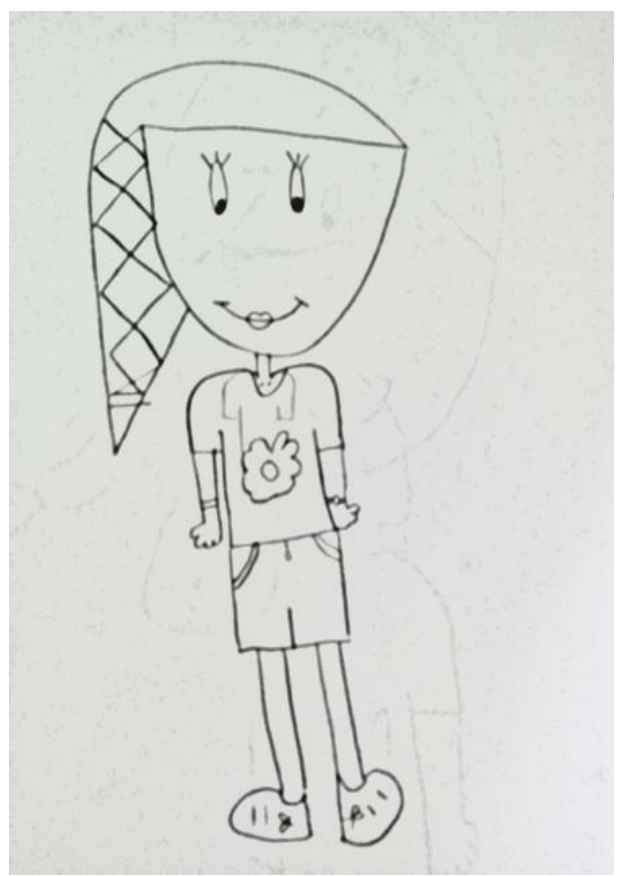

Figura 5. Dibujo de mujer. Niña $5^{\circ} \mathrm{EP}$ (Puntuación bruta test GHDT=35)

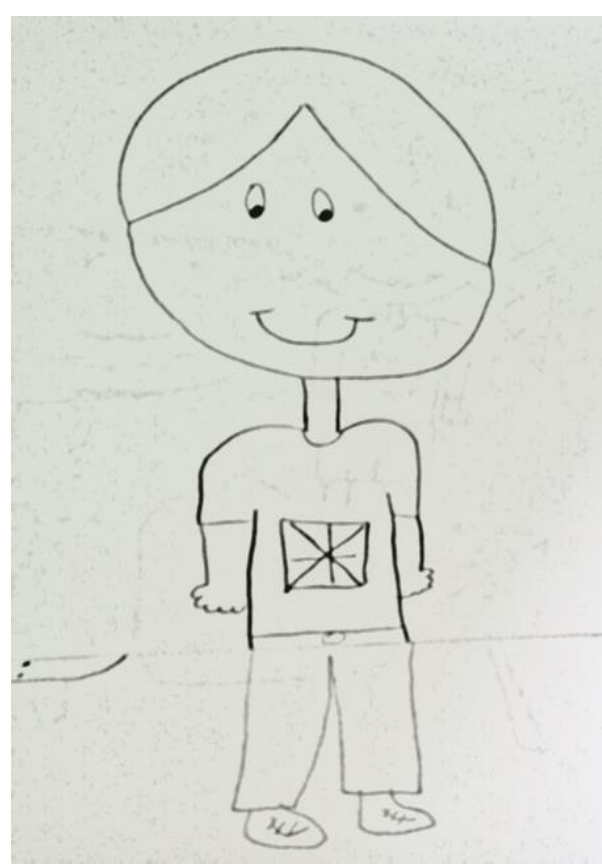

Figura 4. Dibujo de hombre. Niño $4^{\circ} \mathrm{EP}$ (Puntuación bruta test GHDT=25)

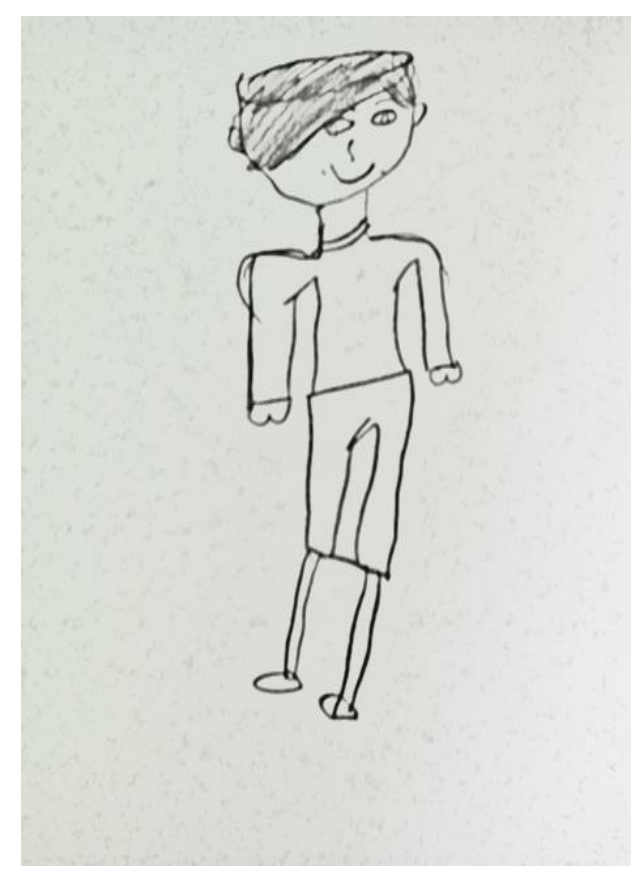

Figura 6. Dibujo de hombre. Niña $5^{\circ}$ EP (Puntuación bruta test GHDT=30) 


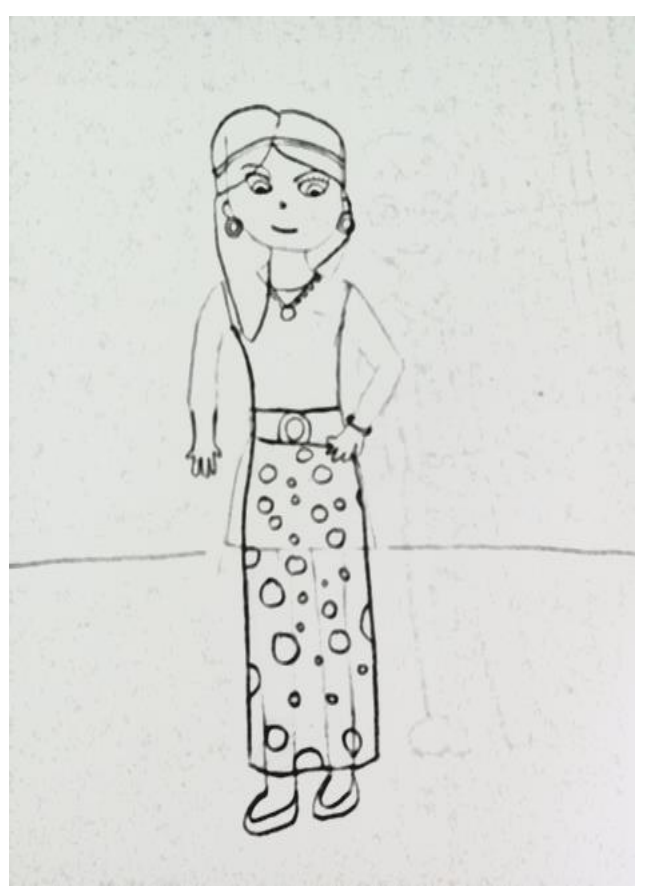

Figura 7. Dibujo de mujer. Niña $6^{\circ} \mathrm{EP}$ (Puntuación bruta test GHDT $=45$ )

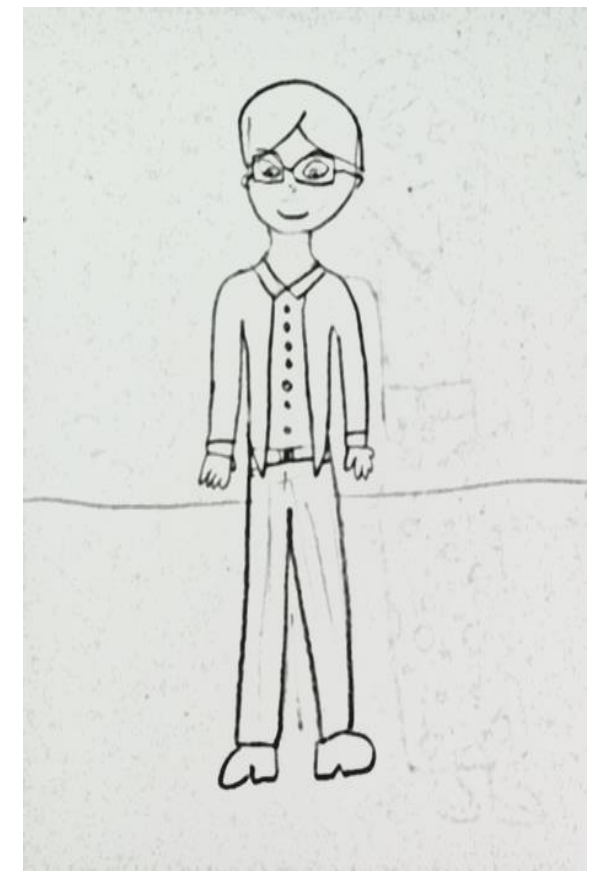

Figura 8. Dibujo de hombre. Niña $6^{\circ} \mathrm{EP}$ (Puntuación bruta test GHDT=41)

\section{Discusión}

El objetivo de este estudio fue determinar la asociación entre el deporte realizado por los padres y el realizado por sus hijos y cómo a su vez la mayor práctica deportiva realizada por el alumnado correspondía con mayores niveles en el rendimiento académico y la habilidad cognitiva de éstos. Se encontraron dos hallazgos principalmente, el primero se determinó que a mayor práctica deportiva por parte de los padres más práctica deportiva por parte del hijos siendo los padres un ejemplo para los más pequeños de la casa. Y un segundo hallazgo que establece que la mayor práctica deportiva del alumnado va relacionada con las dos variables cognitivas estudiadas.

Estudios anteriores comprobaron que una mayor condición física por parte del alumnado se considera un indicador del funcionamiento cognitivo (Hillman et al., 2009). La condición física aeróbica en la infancia está relacionada con la cognición y las diferencias en la estructura regional y función del cerebro (Chaddock, Pontifex, Hillman, \& Kramer, 2011). Una de las explicaciones referida a esta asociación motoracognitiva es la de Wassenberg et al. (2005), que muestra el desarrollo paralelo de rendimiento cognitivo y motor específico en los niños durante el desarrollo normal o retrasado. Algunas estructuras cerebrales específicas, como por ejemplo, los ganglios basales o corteza frontal, y la transmisión de dopamina se deben tanto al rendimiento cognitivo como al motor. Además hay un asociación entre el ejercicio físico, las catecolaminas y la cognición (McMorris, Sproule, Turner, \& Hale, 2011).

En contraposición con lo indicado anteriormente, Best (2010) determina que la repetición de ejercicios aeróbicos disminuye los niveles cognición del alumnado, 
exigiendo que los ejercicios sean complejos para que estos influyan de manera positiva en dicha cognición. Además se puede tener una buena capacidad intelectual y unas buenas aptitudes físicas y, sin embargo, no estar obteniendo un rendimiento adecuado, ante la disyuntiva y con la perspectiva de que el rendimiento académico es un fenómeno multifactorial (Jiménez, 2000).

Esta investigación nos deja patente la idea de que es importante promover cambios legislativos para aumentar las oportunidades de actividad física para los niños en edad escolar, que beneficiarán no sólo a la salud física, sino también, al desarrollo cognitivo y al rendimiento académico. El ejercicio físico puede mejorar aspectos del funcionamiento mental de los niños que son fundamentales para el desarrollo cognitivo (Tomporowski et al., 2008). Sobre este hecho, Lupu (2012) advierte de la necesidad de que la Educación Física se enseñe incluso desde el jardín de infancia, ya que las personas que participan constantemente en clases de Educación Física tienen una capacidad creativa más desarrollada.

El presente estudio tiene varias limitaciones que motivan el trabajo futuro. Una limitación es su diseño transversal en el que se recogen los datos en un determinado momento lo cual puede no darnos fiabilidad absoluta. Atendiendo a esto es idóneo para futuras investigaciones realizar un pre-test y un post-test y cuidar la interpretación de las asociaciones observadas. Son necesarios ensayos controlados, aleatorios y longitudinales para que se presenten pruebas con suficiente causalidad entre la aptitud física y los procesos cognitivos. Por otra parte, el hecho de determinar una de las variables a través de un cuestionario puede aumentar los niveles de subjetividad y obtener respuestas que no son del todo ciertas por parte de los padres. Sin embargo, es casi imposible obtener dicha información mediante otros instrumentos, lo cual nos ha llevado a hacerlo de la forma que se ha expuesto. Otro punto de partida a tener en cuenta en futuras investigaciones, ya que puede ser un aspecto negativo, es que aunque nos hemos centrado en una medida cognitiva específica, los métodos de este estudio, la muestra (tamaño y edad), el diseño del estudio y la medición de la cognición, hacen que sea difícil comparar los resultados con otras investigaciones.

En conclusión, se confirma que una mayor práctica deportiva realizada por los padres se asocia a una mayor práctica realizada por sus hijos. Niveles más altos de práctica deportiva del alumnado se asocian a su vez con un mayor rendimiento académico y una más elevada habilidad cognitiva.

\section{Bibliografía}

Andújar, A. J. C. (2001). La incidencia de la práctica físico-deportiva de los padres hacia sus hijos durante la infancia y la adolescencia. Apunts. Educación física y deportes, 3(65), 100-104.

Botía, A. (2006). Familia y escuela: dos mundos llamados a trabajar en común. Revista de Educación, 339, 119-146. doi: 10.4438/1988-592X-0034-8082-RE 
Casterad, J. Z. (2004). Dimensiones de la condición física saludable: evolución según edad y género dimensions of the healthy physical fitness: Recuperado de https://www.researchgate.net/profile/Javier_Zaragoza/publication/237744436_D imensiones_de_la_condicin_fsica_saludable_evolucin_segn_edad_y_gnero_dim ensions_of_the_healthy_physical_fitness_evolution_for_age_and_gender/links/5 4678e170cf2397f782be9f9.pdf

Chaddock, L., Pontifex, M. B., Hillman, C. H., \& Kramer, A. F. (2011). A review of the relation of aerobic fitness and physical activity to brain structure and function in children. Journal of the International Neuropsychological Society: JINS, 17(6), 975-85. doi: 10.1017/S1355617711000567

Devís, J., y Velert, C. (1992). Nuevas perspectivas curriculares en Educación Física: la salud y los juegos modificados. Recuperado de https://books.google.es/books?hl=es\&lr=\&id=I4oB_7fD0BkC\&oi=fnd\&pg=PA $11 \& \mathrm{dq}=($ Dev\%C3\%ADs,+Peiro,+1992),\&ots=FCSE5blmXD\&sig=Z8pd3xRox 1okQJBdJ_BDUR35dmc

Diamond, a. (2000). Close interrelation of motor development and cognitive development and of the cerebellum and prefrontal cortex. Child Development, 71(1), 44-56. doi: 10.1111/1467-8624.00117

Heinonen, K., Räikkönen, K., Pesonen, A.-K., Kajantie, E., Andersson, S., Eriksson, J. G., ... Lano, A. (2008). Prenatal and postnatal growth and cognitive abilities at 56 months of age: a longitudinal study of infants born at term. Pediatrics, 121(5), e1325-e1333. doi: 10.1542/peds.2007-1172

Hernández, J. G., \& Portolés, A. (2016). Recomendaciones de actividad física y su relación con el rendimiento académico en adolescentes de la Región de Murcia. Retos: nuevas tendencias en educación física, deporte y recreación, (29), 100-104.

Hillman, C. H., Buck, S. M., Themanson, J. R., Pontifex, M. B., \& Castelli, D. M. (2009). Aerobic fitness and cognitive development: Event-related brain potential and task performance indices of executive control in preadolescent children. Developmental Psychology, 45(1), 114-29. doi: 10.1037/a0014437

Janssen, M., Chinapaw, M. J. M., Rauh, S. P., Toussaint, H. M., van Mechelen, W., \& Verhagen, E. A. L. M. (2014). A short physical activity break from cognitive tasks increases selective attention in primary school children aged 10-11. Mental Health and Physical Activity, 7(3), 129-134. doi: 10.1016/j.mhpa.2014.07.001

McMorris, T., Sproule, J., Turner, A., \& Hale, B. J. (2011). Acute, intermediate intensity exercise, and speed and accuracy in working memory tasks: a metaanalytical comparison of effects. Physiology \& Behavior, 102(3-4), 421-8. doi: 10.1016/j.physbeh.2010.12.007 
Navarro, R. E. (2003). El rendimiento académico: concepto, investigación y desarrollo. Revista iberoamericana sobre Calidad, Eficacia Y Cambio en la educación. 1(2) 1-15. doi: 10.15366/reice

Ortega, F. B., Artero, E. G., Ruiz, J. R., España-Romero, V., Jiménez-Pavón, D., Vicente-Rodriguez, G., Castillo, M. J. (2011). Physical fitness levels among European adolescents: the HELENA study. British Journal of Sports Medicine, 45(1), 20-29. doi: 10.1136/bjsm.2009.062679

Soga, K., Shishido, T., \& Nagatomi, R. (2015). Executive function during and after acute moderate aerobic exercise in adolescents. Psychology of Sport and Exercise, 16, 7-17. doi: 10.1016/j.psychsport.2014.08.010

Suárez, Á., y Parra, M. (2005). Actitudes de los padres ante la promoción de la actividad física y deportiva de las chicas en edad escolar. Cuadernos de Psicología Del Deporte, 5(1-2), 174-195.

Tomporowski, P. D., Davis, C. L., Miller, P. H., \& Naglieri, J. a. (2008). Exercise and children's intelligence, cognition, and academic achievement. Educational Psychology Review, 20(2), 111-131. doi: 10.1007/s10648-007-9057-0

Travill, A. L. (2011). Correlation Between Growth and Physical Fitness of Socially Disadvantaged Girls. Growth (Lakeland), 33(3), 9069.

Trudeau, F., Shephard, R.J. (2008). Physical education, school physical activity, school sports and academic performance. Int J Behav Nutrit Physl Activ, 5(1), 5-10. doi: 10.1186/1479-5868-5-10.

Fecha de recepción: 04/04/2016

Fecha de revisión: 07/10/2016

Fecha de aceptación: 09/11/2016 